\title{
Gentiana sect. Chondrophyllae (Gentianaceae) in Australia
}

\author{
L.G. Adams ${ }^{1}$ and J.B. Williams ${ }^{2}$
}

\begin{abstract}
Adams, L.G. ${ }^{I}$ \& Williams, J.B. ${ }^{2}\left({ }^{1}\right.$ Australian National Herbarium, CSIRO, Canberra, Australia 2601; ${ }^{2}$ Botany Department, University of New England, Armidale, Australia 2351) 1988. Telopea 3(2): 167-176. Gentiana sect. Chondrophyllae (Gentianaceae) in Australia. - For the first time sect. Chondrophyllae Bunge is recorded from Australia, being represented by four new species: G. baeuerlenii, G. bredboensis, G. wingecarribiensis and G. wissmannii. A brief history of their discovery is given; the geography of the section, and the features distinguishing it from the purely Southern Hemisphere sections Andicola and Antarctophila Kusn., present in Australasia, are discussed. Full Latin and English descriptions, locality and habitat data (where known), conservation status, and a species key are provided.
\end{abstract}

\section{Introduction}

In March 1887 Wilhelm Baeuerlen, the resident postmaster of the Southern Tablelands town of Bombala, N.S.W., and also a keen amateur naturalist, collected a tiny Gentian on the property 'Quidong', about $20 \mathrm{~km}$ west of the town. He sent it to Ferdinand von Mueller in Melbourne [MEL] for identification; Mueller apparently requested more material and Baeuerlen was able to find a further small quantity (Baeuerlen in herb. MEL, no date, in litt.). The taxon has never been recorded since, which is hardly surprising considering the remoteness of the area from main taxonomic research centres, and the ease of overlooking such a small plant, especially as the corollas no doubt exhibit the photonastic (or thermonastic?) movements (Harder et al. 1965: 380-381; Pringle 1979) characteristic of the genus sensu lato and tend to 'disappear' in dull weather.

After a gap of eighty years two further taxa were discovered, by remarkable coincidence in the same year. One, at first thought to be Baeuerlen's long-lost plant, was found by the senior author south of Jerangle, also in the Southern Tablelands, in June 1967; the other was collected a few months later in Wingecarribee Swamp, east of Moss Vale, N.S.W., by P. Bates. Then, in 1973, a fourth taxon very similar to the latter was found by $H$. Wissmann, this time near the Northern Tablelands town of Ebor, N.S.W.

It is immediately evident that all four taxa belong in Gentiana sect. Chondrophyllae Bunge (1829), as circumscribed by Pringle (1979). None of them can be satisfactorily equated with any previously described, including the 23 species recorded from Papua New Guinea by Royen (1983). However, an affinity with many of the latter appears to be indicated by the lack of basal rosulate leaves in $G$. wingecarribiensis and $G$. wissmannii, a feature also characteristic of the somewhat narrower circumscription of the section for Europe by Tutin (1972). The other two species, $G$. baeuerlenii and $G$. bredboensis, seem to have closer affinities with Eurasian elements of the section as circumscribed by Grossheim (1967) ex descriptio, especially members of his 
series Aquaticae Grossh., characterised by small corollas, obovoid capsules and annual growth habit (but see Marquand 1938, Ho 1985). Until the taxonomic infrastructure of Chondrophyllae is more rigorously defined it would be premature to attempt to place the Australian taxa in a detailed context.

The taxa of sect. Chondrophyllae Bunge are clearly distinct from those purely Southern Hemisphere gentians in Australasia that belong to the sections Andicola and Antarctophila of Kusnezov (1895). These latter, referred to as the 'southern gentians' in a lucid appraisal of this problematical genus sensu lato by Philipson (1972), are currently retained in Gentiana in most New Zealand literature but considered by several workers better placed in the genus Gentianella Moench (in our opinion with some justification, perhaps as a new section). The Chondrophyllae are readily distinguished from the 'southern gentians' by the presence of interpetaline plicae (resulting in a relatively much longer corolla tube), the rigid anthers (not versatile), and the ovarian (as opposed to corolline) nectaries.

Sect. Chondrophyllae is a large, typically montane, and predominantly Northern Hemisphere group. In the Old World it is distributed from Europe (Pyrenees and Alps) through central and eastern Asia to Japan and into Southeast Asia, Indonesia, New Guinea and the Philippines; in the New World, from the Aleutians, Alaska and the Rocky Mountains to Mexico and Central America, and the Andes from Venezuela to Tierra del Fuego (Pringle 1979). The section has not been previously designated for Australia, and its discovery here constitutes a significant extension of range in the southern latitudes of the Old World.

In view of this, it is ironic that all four species fall within Conservation Status (Leigh et al., 1981) of ' $\mathrm{X}$ ' (presumed extinct), ' $\mathrm{E}$ ' (endangered) or ' $\mathrm{V}$ ' (vulnerable). Because of the plants' small stature, viable populations are probably dependent on a stable habitat of short, damp turf of forbs, sedges, grasses and bryophytes, to judge from our field observations. Prior to European settlement this type of habitat was maintained presumably by native herbivores, with negligible amounts of other disturbance. Modifications brought about since by trampling and over-grazing by stock, however, especially in times of drought, together with permanent changes in the watertable as well as other forms of disturbance, have no doubt drastically destabilised habitats. The point has now been reached where all species could be seriously at risk unless management policies geared towards a return to optimum conditions are initiated.

For example, G. wingecarribiensis seems to be restricted exclusively to a subtle micro-habitat zone of sedge/bryophyte tussocks within a fen community. Its long-term survival could depend on the watertable remaining relatively high and fluctuating only within limits of a few centimetres for lengthy, and possibly critical, seasonal periods.

\section{Key to the species}

1 Plants usually with a basal rosette of 2-6 pairs of leaves much larger than the cauline leaves and with more or less smooth margins; corolla white or very pale blue; fruiting gynophore much less than $10 \mathrm{~mm}$ long

$1^{*}$ Plants rarely with a basal rosette, and if so then the leaves not or little larger than the cauline leaves, and the margins clearly scaberulous; corolla sky blue; fruiting gynophore 10-25 mm long 
2 Basal leaves to $8 \mathrm{~mm}$ long, the apices obtuse or shortly mucronate; corollalobes whitish blue inside; plicae 1(-2)-lobed; anthers c. $0.5 \mathrm{~mm}$ long; fruiting gynophore $5 \mathrm{~mm}$ long

1. G. baeuerlenii

2* Basal leaves to $20 \mathrm{~mm}$ long, the apices acute or acuminate, usually mucronate; corolla-lobes pure white inside; plicae lacerate or (1-)2-lobed; anthers c. $1 \mathrm{~mm}$ long; fruiting gynophore $2.5 \mathrm{~mm}$ long

2. G. bredboensis

3 Stem scaberulous above, leafless basal part $10-25 \mathrm{~mm}$ long; fruiting gynophore 15-25 $\mathrm{mm}$ long ......

3. G. wingecarribiensis

$3^{*}$ Stem smooth throughout, leafless basal part $5-10 \mathrm{~mm}$ long; fruiting gynophore $10-15(-20) \mathrm{mm}$ long

4. G. wissmannii

\section{G. baeuerlenii L. Adams, sp. nov.}

[G. quadrifaria auct. non Blume (1826: 847): Mueller (1888a:14, 1888b: 357, 1889: 152).]

Herba annua, erecta, glabra, $2-4 \mathrm{~cm}$ alta. Radix tenuis, simplex. Caulis simplex vel sparsim ramosus, superne scaberulus, basin versus laevis; pars aphylla basalis brevissima vel nulla. Folia basalia (1-)2 paribus, sessilia, late ovata, usque ad $8 \mathrm{~mm}$ longa; apices obtusi vel mucronulati, vix recurvi; margines laeves, tenues, infirme cartilaginei. Folia caulina 3-4 paribus, foliis basalibus similia sed parviora, 4-6 mm longa, superiora marginibus crassioribus. Florae 1-3, singulares, apicales, (4-)5-merae. Calyx anguste infundibuliformis, 4-5 mm longus, (4-)5-lobus; costae anguste alatae; lobi ovati, acuminati, erecti, 1-2.5 mm longi, obscure 3-nerves, membranis intersepalinis connexi. Corolla anguste campanulata, 5-8 mm longa, (4-)5-loba, plicata, "viridula extus, albida caerulescens intus' (fide Muelleri); lobi patentes, acuti, apice $2-3 \mathrm{~mm}$ liberi. Plicae apicibus acuminate 1(-2)-lobae, (albi?), translucentes. Stamina (4-)5, tubo corollae inclusa. Fila c. $4 \mathrm{~mm}$ longa, dimidio inferiore adnata. Antherae c. $0.5 \mathrm{~mm}$ longae. Ovarium stipitatum, subcompressum, alis secus suturas membranaceis angustis. Stylus $1 \mathrm{~mm}$ longus. Stigmata 2, linearia, c. $0.5 \mathrm{~mm}$ longa, recurva, in fructu persistentia. Nectaria rudimentaria, ad ovarium affixa. Gynophorum $1.5 \mathrm{~mm}$ longum, demum in fructu usque ad $5 \mathrm{~mm}$ elongatum. Capsula ovoidea, bivalvis, $3.5 \mathrm{~mm}$ longa. Semina numerosa, 'turgida ovata, minuta, sine appendix; testa subtiliter striolata, pallide fusca' (fide Muelleri, holotypo, MEL). Fig. 1.

TyPUS: Quiedong [Quidong], Southern Tablelands, N.S.W. [Quidong is $18 \mathrm{~km}$ W of Bombala: precise locality of collection unknown but approximately $\left.37^{\circ} \mathrm{S}, 149^{\circ} \mathrm{E}\right], W$. Baeuerlen 462, March 1887 (holo: MEL; iso: NSW).

Habit: annual, erect, glabrous, 2-4 cm high. Taproot: slender, unbranched. Stem: simple or sparsely branched, minutely scaberulous above, smooth below; basal leafless portion very short or absent. Basal leaves: (1-)2 pairs, sessile, broadly ovate, up to $8 \mathrm{~mm}$ long; apices obtuse or very shortly mucronate, scarcely recurved; margins smooth, thin and weakly cartilaginous. Cauline leaves: 3-4 pairs, like the basal leaves but smaller, 4-6 $\mathrm{mm}$ long, the upper with thicker margins. Flowers: 1-3, solitary, terminal, (4-)5-merous. Calyx: narrowinfundibuliform, 4-5 mm long, (4-)5-lobed; ribs narrowly winged; lobes ovate, acuminate, erect, 1-2.5 mm long, obscurely 3-veined, connected by intersepaline membranes. Corolla: narrow-campanulate, 5-8 $\mathrm{mm}$ long, (4-)5-lobed, plicate, 'greenish outside, whitish-blue inside' (fide Mueller); lobes spreading, acute, the 


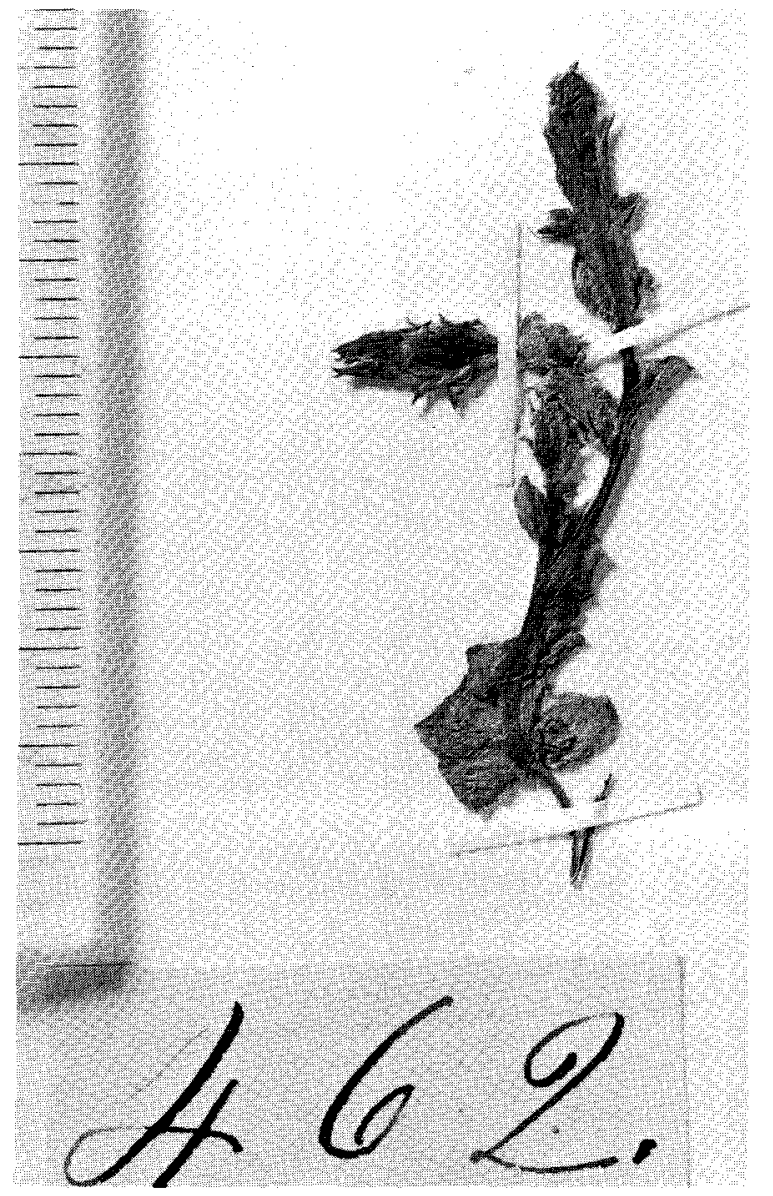

Fig. 1. Gentiana baeuerlenii (from holotype). Scale in $\mathrm{mm}$.

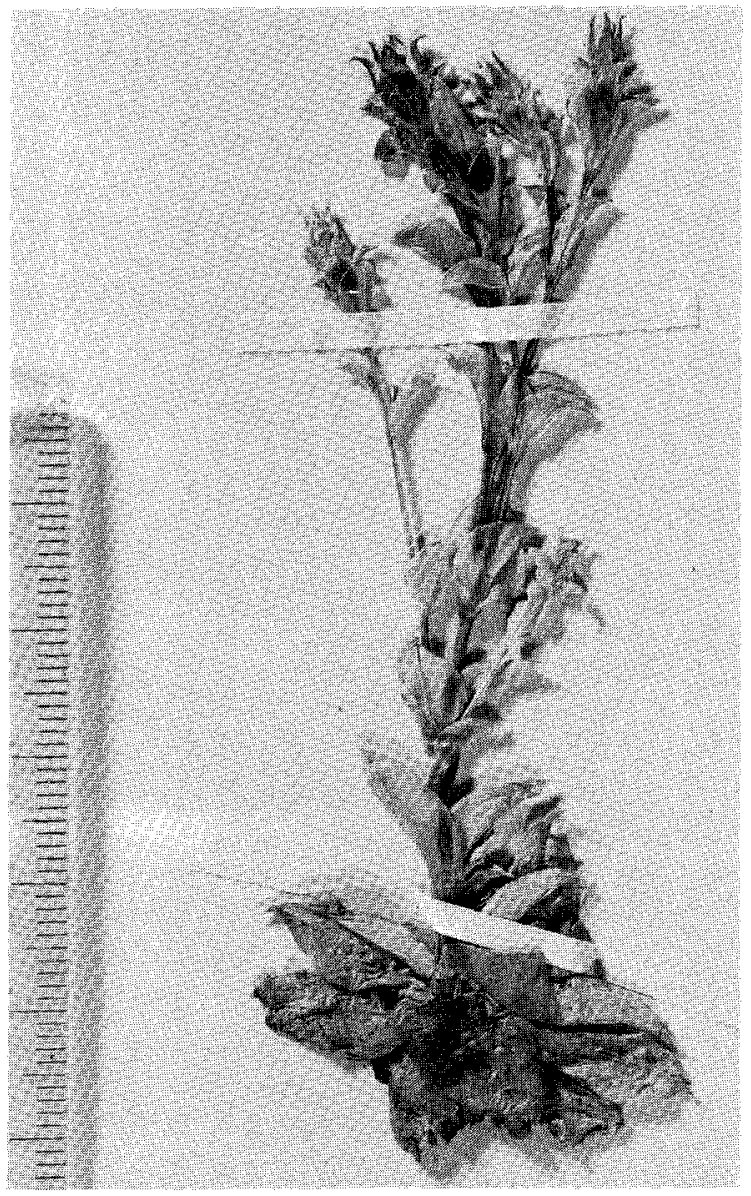

$\Xi$

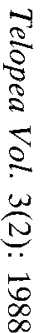


upper 2-3 mm free. Plicae: with apices acuminately 1(-2)-lobed, (white?), translucent. Stamens: (4-)5, enclosed in corolla-tube. Filaments: c. $4 \mathrm{~mm}$ long, adnate in lower half. Anthers: c. $0.5 \mathrm{~mm}$ long. Ovary: stipitate, sub-compressed, with narrow membranous wings along sutures. Style: $1 \mathrm{~mm}$ long. Stigmas: 2, linear, c. $0.5 \mathrm{~mm}$ long, recurved, persistent in fruit. Nectaries: rudimentary, ovarian. Gynophore: $1.5 \mathrm{~mm}$ long, elongating to $5 \mathrm{~mm}$ in fruit. Capsule: ovoid, 2-valved, $3.5 \mathrm{~mm}$ long. Seeds: numerous, 'turgid-ovate, minute, without an appendage; testa finely striolate, pale brown' (fide Mueller, holotype, MEL).

NOTES: 'Flowers closed up [at time of gathering?], white with bluish tinge' (fide Baeuerlen, holotype, MEL). Baeuerlen's plant is generally similar in size and appearance and obviously closely related to both $G$. quadrifaria B1. and $G$. aquatica L., but these species have significant differences. G. quadrifaria, from Java, has ovate leaves with conspicuously acuminate-aristate apices. $G$. aquatica, widespread in central and eastern Asia, differs in its lax, basally multibranching habit and narrowly oblong-lanceolate cauline leaves. Another related Asiatic species is G. pseudo-aquatica Kusn. but this is also basally multibranched and the cauline leaves are strongly spathulate.

A label with a fragment of Baeuerlen's collection in the NSW herbarium states: 'Quiedong nr. Bombala NSW, $W$. Baeuerlen $n r$. 462' in an unknown hand. The MEL sheet bears two labels, both carrying 'Nro. 462' in Baeuerlen's hand and annotated by Mueller as ' $G$. quadrifaria Bl.', although on one he also states 'near G. aquatica L.'. However, while one label mentions 'Quiedong' (in Baeuerlen's writing), on the other Mueller has written 'Genoa'! The latter would seem to be the explanation for this plant later being listed for Victoria (Mueller $1888 \mathrm{a}, \mathrm{b} ; 1889)$. We can find no evidence that Wilhelm Baeuerlen ever collected in the Genoa area of East Gippsland, Victoria, and although it is not inconceivable for the plant to occur there we consider that without further evidence this 'record' is an error.

HABITAT: Unknown, but presumably damp places in short turf.

CONSERVATION STATUS: 1 ? X. This species is still known only from the type collection, despite some searching by the senior author of likely sites on the Quidong property. In view of the general remoteness of the area and the diminutive habit of this easily overlooked plant, it is too soon to presume the species extinct; every opportunity should be taken to search for it in suitable habitats of the Delegate River catchment.

\section{G. bredboensis L. Adams, sp. nov.}

Herba annua vel ?biennis, erecta, glabra, $2-9 \mathrm{~cm}$ alta. Radix tenuis, plerumque ramosa. Caulis plerumque multiramosus, raro simplex, scaberulus; pars aphylla basalis brevissima. Folia basalia 3-6 paribus, sessilia, late ovata, usque ad $20 \mathrm{~mm}$ longa; apices acuti vel acuminati, plerumque mucronati, recurvi; margines laeves vel sub-scaberulus, crassiusculis, cartilagineis. Folia caulina 3-6 paribus, foliis basalibus similia sed sursum gradatim parvioria et marginibus crassioribus, 6-15 mm longa. Florae 1-6, singulares, apicales, (4-)5merae. Calyx anguste infundibuliformis, 5-8 mm longus, (4-)5-lobus; costae anguste alatae; lobi ovati, acuti, erecti, carinati, 2.5-3.5 $\mathrm{mm}$ longi, 1-nerves; membranis intersepalinis connexi. Corolla anguste campanulata, $8-10 \mathrm{~mm}$ longa, (4-)5-loba, plicata, costis sub-roseis extus, candida intus; lobi patentes, acuti, apice 2-4 $\mathrm{mm}$ liberi. Plicae apicibus laceratae vel irregulariter (1-)2-lobae, 
albotranslucentes. Stamina 5, tubo corollae inclusa. Fila 3-5 mm longa, dimidio inferiore adnata. Antherae c. $1 \mathrm{~mm}$ longae. Ovarium stipitatum, subcompressum, alis secus suturas membranaceis. Stylus $0.5 \mathrm{~mm}$ longus. Stigmata 2, linearia, c. $3 \mathrm{~mm}$ longa, recurva, in fructu persistentia. Nectaria rudimentaria, ovarium affixa. Gynophorum $1.5 \mathrm{~mm}$ longum, demum in fructu usque ad $2.5 \mathrm{~mm}$ elongatum. Capsula oblonge ovoidea, bivalvis, 5-6 mm longa. Semina numerosa, oblique ovoidea, $0.6 \mathrm{~mm}$ longa, sine appendice; testa reticulata striolata, brunnea. Fig. 2.

TYPUS: c. 7 miles S [11 km SSE] of Jerangle, Southern Tablelands, N.S.W., alt. $930 \mathrm{~m}$, $35^{\circ} 58^{\prime} \mathrm{S} 149^{\circ} 25^{\prime} \mathrm{E}$, grid ref. GA 171175, Pullen \& Adams 4427, 3.xii.1971 (holo: CANB; iso: $\mathrm{K}, \mathrm{L}, \mathrm{MEL}, \mathrm{NE}, \mathrm{NSW}, \mathrm{US}$ ).

Habit: annual or ?biennial, erect, glabrous, 2-9 cm high. Taproot: slender, usually branched. Stem: usually many-branched, rarely simple, scaberulous; basal leafless portion very short. Basal leaves: 3-6 pairs, sessile, broadly ovate, up to $20 \mathrm{~mm}$ long; apices acute or acuminate, usually mucronate, recurved; margins smooth or sub-scaberulous, moderately thick and cartilaginous. Cauline leaves: 3-6 pairs, like basal leaves but becoming smaller upward and with thicker margins, 6-15 mm long. Flowers: 1-6, solitary, terminal, (4-)5-merous. Calyx: narrow-infundibuliform, 5-8 mm long, (4) 5-lobed; ribs narrowly winged; lobes ovate, acute, erect, keeled, 2.5-3.5 mm long, 1-veined, connected by intersepaline membranes. Corolla: narrow-campanulate, 8-10 mm long, (4-)5lobed, plicate, pinkish-ribbed outside, pure white inside; lobes spreading, acute, the upper 2-4 mm free. Plicae: with the apices lacerate or irregularly (1-)2lobed, white, translucent. Stamens: 5, enclosed in corolla-tube. Filaments: 3-5 mm long, adnate in lower half. Anthers: c. $1 \mathrm{~mm}$ long. Ovary: shortly stipitate, compressed, with narrow membranous wings along sutures. Style: $0.5 \mathrm{~mm}$ long. Stigmas: 2, linear, c. $1.5 \mathrm{~mm}$ long, recurved, persistent in fruit. Nectaries: rudimentary, ovarian. Gynophore: $1.5 \mathrm{~mm}$ long, shortly elongating up to $2.5 \mathrm{~mm}$ in fruit. Capsule: oblong-ovoid, 2-valved, 5-6 mm long. Seeds: numerous, obliquely ovoid, $0.6 \mathrm{~mm}$ long, without appendages; testa reticulatestriolate, brown.

NOTES: Flowers only opening in direct sunlight.

HABITAT: Margins of very wet seepage slopes in sheep pasture on granitic sandy soil; closely grazed turf amongst Baeckea-Leptospermum thickets.

Conservation Status: $2 \mathrm{~V}$. This species is known only from the type locality, on private property, over a distance of a few hundred metres along a small tributary of the Bredbo River. Many similar pockets of this type of habitat on granitic soils probably exist within the Bredbo River catchment.

Specimens Examined: New South Wales: Southern Tablelands: c. 7 miles S. $[11 \mathrm{~km}$ SSE.] of Jerangle, Adams 1886A, 15.vi.1967 (CANB); Adams et al: 2598, 27.viii.1971 (CANB).

\section{G. wingecarribiensis $L$. Adams, sp. nov.}

Herba annua, erecta, glabra, 4-9 cm alta. Radix tenuis, simplex. Caulis simplex vel sparsim ramosus, superne scaberulus basin versus laevis, valde rubello-suffusus; pars aphylla basalis $1-2.5 \mathrm{~cm}$ longa. Folia basalia nulla. Folia caulina 4-7 paribus, sessilia, late vel oblonge ovata, carinata, $3-8 \mathrm{~mm}$ longa; apices acuti vel obtusi, mucronati, plus minusve recurvi; margines cartilaginei, scaberuli. Florae 1-6, singulares, apicales, (4-)5-merae. Calyx anguste 
infundibuliformis, 6-10 mm longus, (4-)5-lobus; costae exalatae; lobi lanceolati, acuti, erecti, 2-4 mm longi, obscure 3-nerves; membranis intersepalinis connexi. Corolla anguste campanulata, 10-17 mm longa, (4-)5-loba, plicata, virello-costa extus, caerulea intus; lobi patentes, acuti vel acuminati, apice $2-3 \mathrm{~mm}$ liberi. Plicae apicibus laceratae vel irregulariter 1-2-lobae, caeruleae, translucentes. Stamina 5, tubo corollae inclusa. Fila 3-5 $\mathrm{mm}$ longa, dimidio inferiore adnata. Antherae c. $1 \mathrm{~mm}$ longae. Ovarium stipitatum, subcompressum, alis secus suturas membranaceis. Stylus nullus. Stigmata 2, linearia, c. $3 \mathrm{~mm}$ longa, recurva, in fructu persistentia. Nectaria rudimentaria, ovario affixa. Gynophorum 2-3 mm longum, demum in fructu ad $15-25 \mathrm{~mm}$ elongatum. Capsula late obovoidea, bivalvis, 4-5 mm longa. Semina numerosa, oblique ellipsoidea fusiformia, $0.3 \mathrm{~mm}$ longa, sine appendice; testa reticulata striolata, pallide brunnea, translucens. Fig. 3.

TYPUS: Wingecarribee Swamp, c. $9.6 \mathrm{~km}$ W of Robertson, Central Tablelands, N.S.W., alt. c. $670 \mathrm{~m}, 34^{\circ} 34^{\prime} \mathrm{S} 150^{\circ} 31^{\prime} \mathrm{E}$, grid ref. KG 718712, Pullen 4999, 3.x.1973 (holo: CANB; iso: NSW).

Habit: annual, erect, glabrous, 4-9 cm high. Taproot: slender, unbranched. Stem: simple or sparsely branched, minutely scaberulous above, smooth below, strongly reddish-tinged; basal leafless portion $1-2.5 \mathrm{~cm}$ long. Basal leaves: absent. Cauline leaves: 4-7 pairs, sessile, broad- or oblong-ovate, keeled, 3-8 mm long; apices acute or obtuse, mucronate, more or less recurved; margins cartilaginous, scaberulous. Flowers: 1-6, solitary, terminal, (4-)5-merous. Calyx: narrow-infundibuliform, 6-10 mm long, (4-)5-lobed; ribs not winged; lobes lanceolate, acute, erect, 2-4 mm long, obscurely 3-veined; intersepaline membranes present. Corolla: narrow-campanulate, 10-17 mm long, (4-)5-lobed, plicate, greenish-ribbed outside, sky blue inside; lobes spreading, acute or acuminate, the upper $2-3 \mathrm{~mm}$ free. Plicae: with apices lacerate or irregularly 1-2-lobed, pale blue, translucent. Stamens: 5, enclosed in corolla tube. Filaments: 3-5 mm long, adnate in lower half. Anthers: c. $1 \mathrm{~mm}$ long. Ovary: stipitate, subcompressed, with membranous wings along sutures. Style: absent. Stigmas: 2, linear, c. $3 \mathrm{~mm}$ long, recurved, persistent in fruit. Nectaries: rudimentary, ovarian. Gynophore: $2-3 \mathrm{~mm}$ long, greatly elongating to $15-25 \mathrm{~mm}$ in fruit. Capsule: broadly obovoid, 2-valved, 4-5 mm long. Seeds: numerous, obliquely ellipsoid-fusiform, $0.3 \mathrm{~mm}$ long, without appendages; testa reticulatestriolate, pale brown, translucent.

NOTES: Corolla opening only in bright sunlight.

HABITAT: Sphagnum hummocks and small open patches in dense Juncus/sedge community, c. $15 \mathrm{~cm}$ above watertable. Not seen in adjoining, floristically different communities, or in wetter areas.

CONSERVATION STATUS: 2E. This species is known only from the type locality, which is on private property. The habitat site, although relatively large, is threatened by permanent flooding and peat mining; no other similar sites are known in the area.

Specimens Examined: New South Wales: Central Tablelands: Pullen 4999, 3.x.1973 (CANB, NSW); P. Bates s.n., 15.ix.1967 (NSW 142611); Johnson \& Briggs s.n., 18.ix.1967 (NSW 142612); Adams et al. 2606, 26.x.1971 (CANB). 


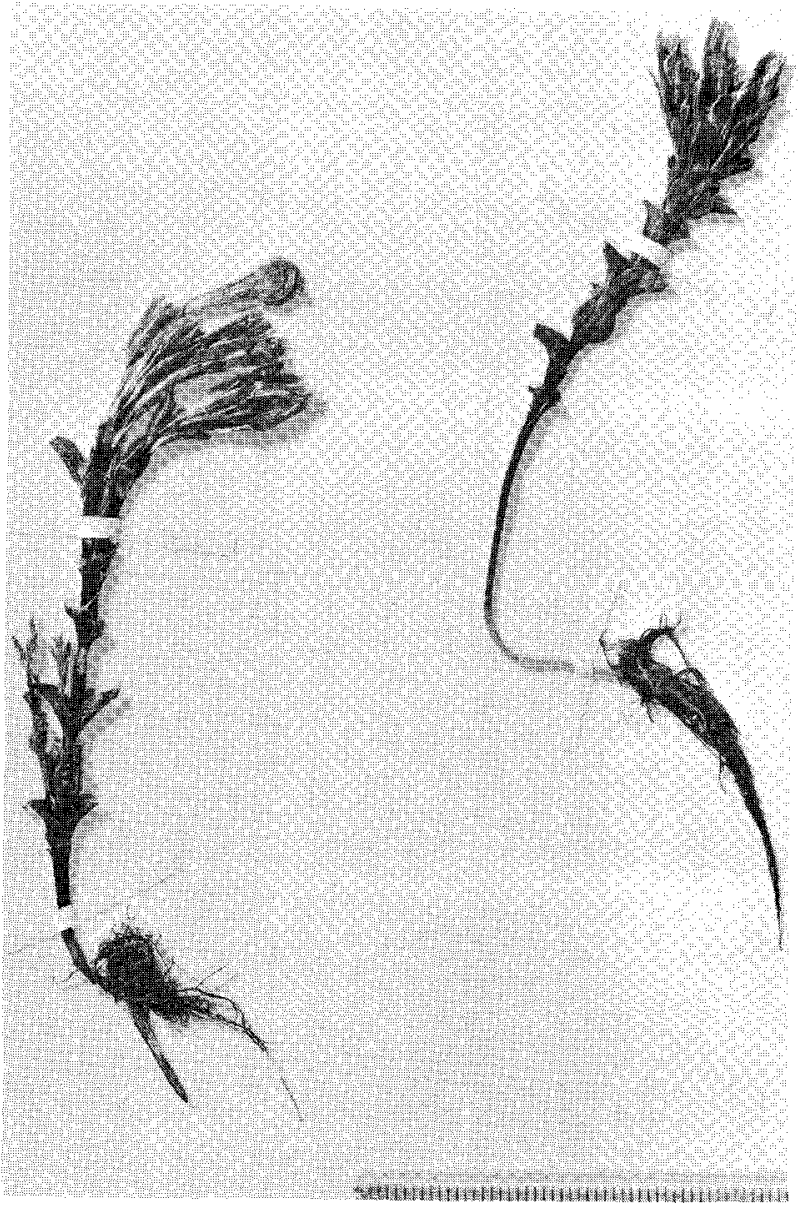

Fig. 3. Gentiana wingecarribiensis (from holotype). Scale in $\mathrm{mm}$.

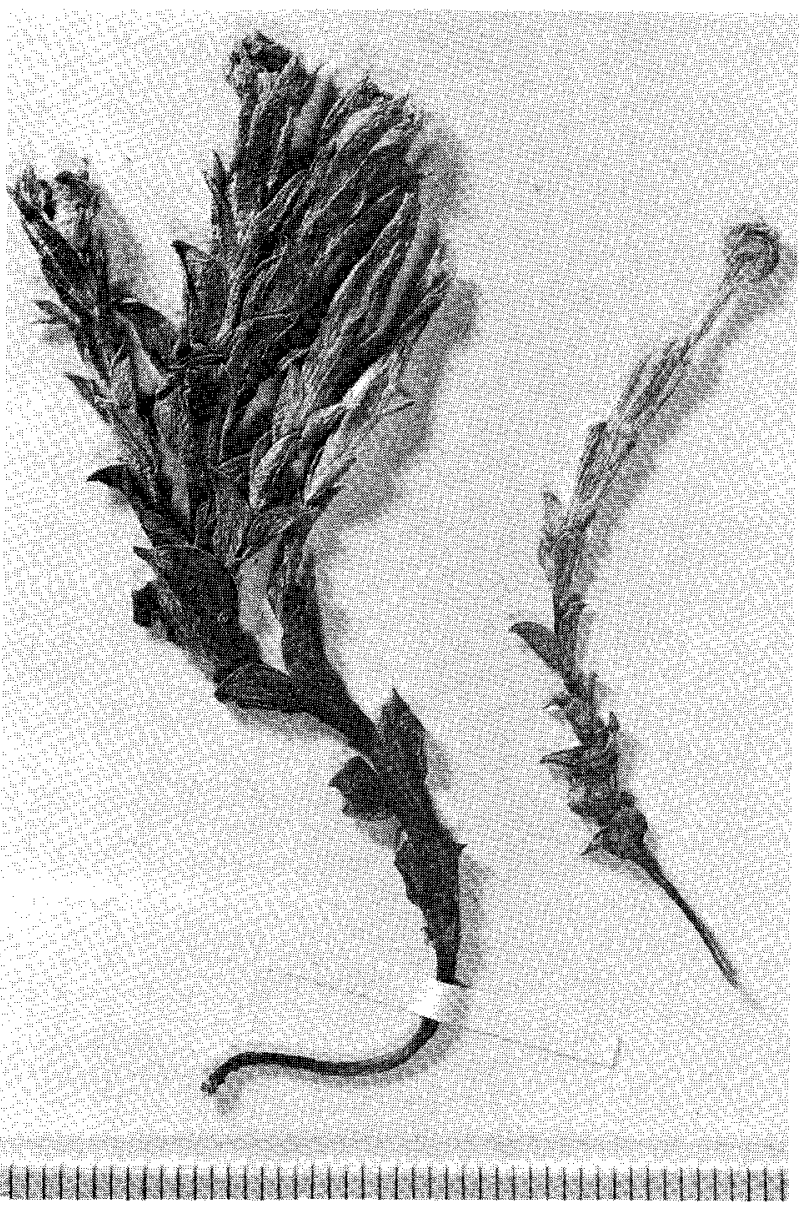




\section{G. wissmannii J.B. Williams, sp. nov.}

Herba annua, erecta, glabra, 3-8 cm alta. Radix tenuis, simplex. Caulis simplex vel 2-4-ramosus, laevis, valde rubello-suffusus; pars aphylla basalis 0.5-1 cm longa. Folia basalia nulla; (folia caulina inferiora plantarum brevissimocaulium sub-rosulata). Folia caulina 3-10 paribus, sessilia, ovata vel oblonge ovata, carinata, 4-10 mm longa; apices acuti vel obtusi, plus minusve recurvi; margines cartilaginei, scaberuli. Florae 1-8, singulares, ramis brevissimis apicales, (4-)5-merae. Calyx anguste infundibuliformis, 8-11 $\mathrm{mm}$ longus, (4-)5lobus; costae exalatae; lobi oblonge ovati vel lancelolati, acuti, erecti, 3-4 mm longi, obscure 3-nerves, membranis intersepalinis connexi. Corolla anguste campanulata, 8-15 mm longa, (4-)5-loba, plicata, virello-costa extus, caerulea intus; lobi patentes, acuti vel breviter acuminati, apice $2-2.5 \mathrm{~mm}$ liberi. Plicae apicibus laceratae vel irregulariter 2-3-lobae, caeruleae, translucentes. Stamina 5 , tubo corollae inclusa. Fila $4-5 \mathrm{~mm}$ longa, dimidio inferiore adnata. Antherae (0.7-)1 mm longae. Ovarium breviter stipitatum, compressum, alis secus suturas membranaceis angustis. Stylus nullus. Stigmata 2(-3), linearia, $2.5 \mathrm{~mm}$ longa, recurva, in fructu persistentia. Nectaria rudimentaria, ovario affixa. Gynophorum c. $2 \mathrm{~mm}$ longum, demum in fructu ad 10-15(-20) $\mathrm{mm}$ elongatum. Capsula obovoidea, 2(-3)-valvis, 5-6 mm longa. Semina numerosa, ellipsoidea fusiformia, $0.3 \mathrm{~mm}$ longa, sine appendice; testa reticulata striolata, cinnamomea, translucens. Fig. 4.

TYPus: Ebor-Armidale road, $1.6 \mathrm{~km} \mathrm{E}$ of Yooroonah, $0.6 \mathrm{~km} \mathrm{E}$ of Oaky Creek, Northern Tablelands, N.S.W., alt. $1100 \mathrm{~m}, 30^{\circ} 30^{\prime} \mathrm{S} 152^{\circ} 16^{\prime} \mathrm{E}$, grid ref. MM 290257 , J.M.B. Smith 221, 2.xi.1976 (holo: CANB; iso: NE).

Habit: annual, erect, glabrous, $3-8 \mathrm{~cm}$ high. Taproot: slender, unbranched. Stem: simple or 2-4-branched, smooth, strongly reddish-tinged; basal leafless portion $0.5-1 \mathrm{~cm}$ long. Basal leaves: absent; (lower cauline leaves of very shortstemmed plants sub-rosulate). Cauline leaves: 3-10 pairs, sessile, ovate to oblong-ovate, keeled, 4-10 mm long; apices acute or obtuse, more or less recurved; margins cartilaginous, scaberulous. Flowers: $1-8$, solitary, terminal on very short branches, (4-)5-merous. Calyx: narrow-infundibuliform, $8-11 \mathrm{~mm}$ long, (4-)5-lobed; ribs not winged; lobes oblong-ovate, lanceolate, acute, erect, 3-4 mm long, obscurely 3-veined; intersepaline membranes present. Corolla: narrow-campanulate, 8-15 mm long, (4-)5-lobed, plicate, greenish-ribbed outside, sky blue inside; lobes spreading, acute or short-acuminate, the upper 2-2.5 mm free. Plicae: with apices lacerate or irregularly 2-3-lobed, pale blue, translucent. Stamens: 5, enclosed in corolla-tube. Filaments: 4-5 mm long, adnate in lower half. Anthers: (0.7-)1 mm long. Ovary: shortly stipitate, compressed, with narrow membranous wings along sutures. Style: absent. Stigmas: 2(-3), linear, $2.5 \mathrm{~mm}$ long, recurved, persistent in fruit. Nectaries: rudimentary, ovarian. Gynophore: c. $2 \mathrm{~mm}$ long, greatly elongating to 10-15(-20) $\mathrm{mm}$ in fruit. Capsule: obovoid, 2(-3)-valved, 5-6 mm long. Seeds: numerous, ellipsoid-fusiform, $0.3 \mathrm{~mm}$ long, without appendages; testa reticulatestriolate, orange-brown, translucent.

NOTES: Flowers opening only in bright sunlight.

HABITAT: Margins of Leptospermum/Restio/Sphagnum swamps, usually in closely-grazed turf (cattle or kangaroos).

CONSERVATION STATUS: $2 \mathrm{~V}$ or 2?C. This species is recorded from the general area between Round Mountain and Ebor; most of the known sites appear to be on private property, but the plant probably also occurs in the adjacent Cathedral Rock National Park. 
Specimens Examined: New South Wales: Northern Tablelands: Round Mountain Area, 50 miles [80 km by road] $\mathrm{E}$ of Armidale, H. Wissmann s.n., 14.x.1973 (CANB, NSW); slopes SE of Cathedral Rocks, near Ebor, J.M.B. Smith 33, 25.ix.1974 (CANB, NE), Kangaroo Creek valley, c. $6 \mathrm{~km}$ E of Round Mountain, J.M.B. Smith \& G.S. Hope 218, 21.x.1976 (CANB, NE), J.M.B. Smith 219, 2.xi.1976 (CANB, NE); Bullock Creek, Armidale-Ebor road, $10 \mathrm{~km} \mathrm{SW}$ of Ebor, J.M.B. Smith 220, 2.xi.1976 (CANB, NE); Armidale-Ebor road, $0.5 \mathrm{~km}$ E of Yooroonah, J.M.B. Smith 222, 4.xi.1976 (CANB, NE); Kangaroo Creek, Ebor-Guyra road, $6 \mathrm{~km}$ W of Ebor, J.M.B. Smith 224, 4.xi.1976. (CANB, NE); Sandy Creek, $10 \mathrm{~km}$ W of Ebor, J.M.B. Smith 225, 4.xi.1976 (CANB, NE).

\section{Acknowledgements}

We wish to record our appreciation of help from the following: Dr Barbara Briggs (NSW), for originally bringing the existence of the Wingecarribee site to our attention; Dr Jeremy M.B. Smith (NE), for his special effort in making extensive collections from the Ebor area; the late Dr Andrew Kanis (CANB), for his painstaking checking of the Latin descriptions and, together with Dr Judy West and Mr Max Gray (CANB), for many useful suggestions; and the Directors of the K, MEL and NSW herbaria, for loans of material.

\section{References}

Blume, C.L. (1826) 'Bijdragen tot de Flora van Nederlandsch Indië (Lands Drukkerij: Batavia).

Bunge, A.A. (1829) Conspectus generis Gentianae imprimis specierum rossicarum: 1824. Nouv. Mem. Soc. Imp. Naturalistes Moscou 1: 207.

Grossheim, A.A. (1967) Gentiana. In V.L. Komarov (ed.), 'Flora of the U.S.S.R.' [English transl.] Vol. 18: 396-456 (Israel Program for Sci. Translations: Jerusalem).

Harder, R., et al. (1965) 'Strasburger's Textbook of Botany' (Longmans: London).

Ho, T.-N. (1985) A study of the genus Gentiana of China (IV). Bull. Bot. N.E. For. Univ. 5(4): 1-22.

Kusnezov, N. (1895) Gentiana. Pp. 80-86 in A. Engler \& K. Prantl (eds), 'Die Naturl. Pflanzenfam. 4(2)' (W. Engelmann: Leipzig).

Leigh, J., Briggs, J., \& Hartley, W. (1981) 'Rare or Threatened Australian Plants'. A.N.P.W.S. Special Publication No. 7 (Govt Printer: Canberra).

Marquand, C.V.B. (1938) The Gentians of China. Kew Bull. 1937: 134-180.

Mueller, F. (1888a) Enumeration of Victorian Plants, Suppl. Victorian Naturalist 5(1): 14-16.

Mueller, F. (1888b) 'Key to System of Victorian Plants 1' (Govt Printer: Melbourne).

Mueller, F. (1889) 'Second Census of Australian Plants' (Govt Printer: Melbourne).

Philipson, W.R. (1972) The generic status of the Southern Hemisphere gentians. Advances Pl. Morphol. 1972: 417-422.

Pringle, J.S. (1979) Taxonomy and distribution of Gentiana (Gentianaceae) in Mexico and Central America, II. Sect. Chondrophyllae. Sida 8(1): 14-33.

Royen, P. van (1983) Gentianaceae. Pp. 2793-2846 in "The Alpine Flora of New Guinea'. Vol. 4 (J. Cramer: Vaduz).

Tutin, T.G. (1972) Gentiana. In T.G. Tutin et al. (eds), 'Flora Europaea'. Vol. 3: 59-63 (Cambridge Univ. Press: Cambridge).

Manuscript received 10 July 1986

Manuscript accepted 23 January 1987 